INNOVATIONS IN PRIMARY CARE

\title{
Expanding the Use of Botulinum Toxin in Primary Care for Chronic Migraine
}

\author{
Blake Busey, DO \\ Ann Fam Med 2019;17:85. https://doi.org/10.1370/afm.2343.
}

\section{THE INNOVATION}

In 2017, the Neurology Department at William Beaumont Medical Center (WBAMC) suffered a loss of clinicians, leaving many patients unable to receive quarterly botulinum toxin injections for chronic migraine headaches. The author developed a program to train and credential primary care clinicians in this specialty procedure.

\section{WHO \& WHERE}

Department of Primary Care of WBAMC, Fort Bliss, Texas.

\section{HOW}

Primary Care and Neurology Departments partnered to train and credential primary care clinicians in botulinum toxin injection for chronic migraine. Chronic migraine is an indication for botulinum toxin therapy shown effective in the Phase 3 REsearch Evaluating Migraine Prophylaxis Therapy (PREEMPT 2) trial ${ }^{1}$ and confirmed in the Chronic Migraine OnabotulinuMtoxinA Prolonged Efficacy open Label (COMPEL) trial ${ }^{2}$ as well as a Cochrane review ${ }^{3}$ with reduction in headache days. The author served as the project champion and developed a 5-component project and curriculum to train primary care clinicians (physicians, physician assistants, and nurse practitioners) within all adult primary care clinics.

The first component was personal development. The author obtained didactic training from Allergan followed by procedural training from a credentialed clinician. The credentials and pharmacy and therapeutics committees granted the author privileges and prescriptive authority, respectively. The author developed standard operating procedures with medication stewardship guidance, a medication supply chain with pharmacy, documentation templates, and a preliminary curriculum.

The second phase was testing and revision. The clinic was

Conflicts of interest: author reports none.

\section{Corresponding author}

Blake Busey, DO

Texas Tech University Health Sciences Center El Paso

Department of Family Medicine - Transmountain,

2000 B, 4th floor

El Paso, Texas 79911

dr.busey.omt@gmail.com used as the prototype for testing and revising the curriculum. The author trained all nursing support on reconstitution, documentation, and coding requirements. For the first month, the team revised the workflow, documentation and coding, and training materials through rapid Plan-Do-Check-Act cycles before initiating training of other clinicians.

The third phase was implementing the curriculum in training and credentialing clinicians. Outlying clinic clinicians attended didactic training before rotating through a procedural day. Over 6 months, the project credentialed 15 clinicians in all 6 outlying medical homes.

The fourth phase was the implementation phase. Trained primary care clinicians scheduled chronic migraine procedures within our system, including those potentially lost to the network. The process recaptured revenue through reduced referral costs, relative value units (RVUs) for the procedure, and medication costs.

The final phase was maintenance and sharing. Other subject matter experts were identified as trainers within each medical home. Additionally, 50 physicians at the 2018 Uniformed Services Academy of Family Physicians national conference were trained through a workshop incorporating this project and didactic training.

\section{LEARNING}

This curriculum was developed out of necessity and was a continuous learning experience. Many patients with chronic migraine have concomitant occipital neuralgia leading to further training on occipital nerve blocks. There is a high potential for botulinum toxin diversion so a 2-person verification of proper use and disposal is recommended. Despite strict guidelines, the temptation to expedite the clinical process to qualify for this procedure came to light. Secondarily, retraining and disciplinary action for clinicians that did not adhere to appropriateness criteria was developed. Subsequent iterations of training focused on appropriateness criteria and consequences of nonadherence before initiating procedure. Expansion of this program to satellite sites was met with resistance from specialty clinicians and hospital systems. Changing this paradigm is a diplomatic and administratively tiresome process. Developing and maintaining professional relationships is key to success. The phases of the project described within this manuscript can be adapted to many other specialty procedures in an underserved community or region.

Supplemental appendix, affiliations, key words, submitted dates, acknowledgments, and references available at http://www.AnnFamMed.org/content/17/1/85/suppl/DC1/. 